\title{
A Collaborative See-through Display Supporting On-demand Privacy
}

David Lindlbauer ${ }^{1}$, Toru Aoki ${ }^{2}$, Anita Höchtt ${ }^{3}$, Yuji Uema ${ }^{2}$, Michael Haller ${ }^{3}$, Masahiko Inami ${ }^{2}$, Jörg Müller ${ }^{1,4}$

${ }^{1}$ Quality and Usability Lab, Telekom Innovation Laboratories, Technical University Berlin, Germany

${ }^{2}$ Keio University, Yokohama City, Kanagawa, Japan

${ }^{3}$ Media Interaction Lab, University of Applied Sciences Upper Austria, Hagenberg, Austria

${ }^{4}$ Alexander von Humboldt Institute for Internet and Society, Berlin, Germany

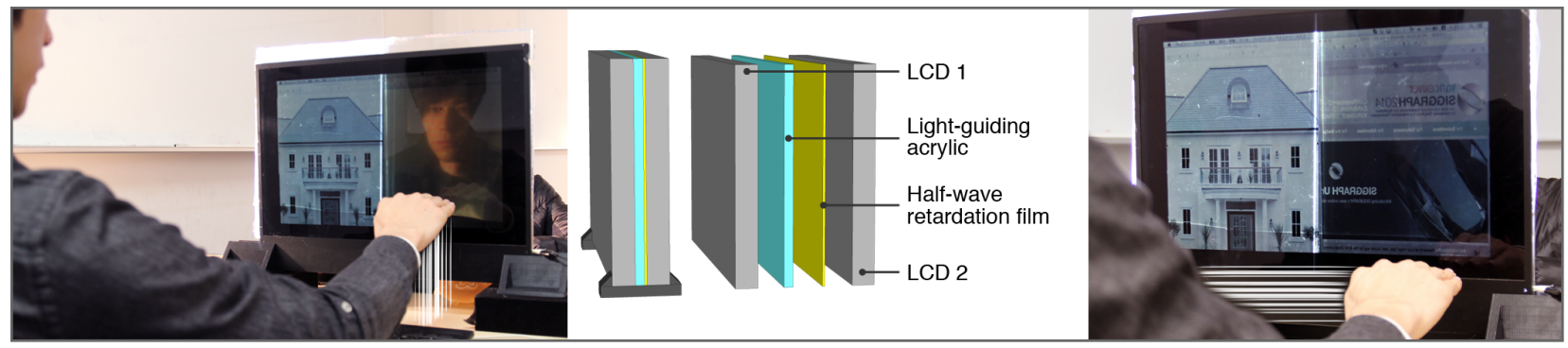

Figure 1: Our display system provides continuous transparency control by using a light-guiding acrylic with edge-mounted LEDs and a

half-wave retardation film to adjust polarization (center). Users can effortlessly and instantly communicate with their co-workers by turn-

ing the display transparent (left), or share information like websites by flipping screen content (right) with simple hand gestures.

\section{Introduction}

Office workers spend a majority of their time in front of their computer display. Therefore the display becomes a constant visual barrier and a barrier for communication. Even workers sitting across a table have to make a high effort to communicate by either standing up or walking around the table to see each other. Transparent displays have a high potential for improving this co-located face-to-face collaboration and information sharing by offering the unique ability to see screen content as well as the environment behind the display. However, with users and their screen content being always visible to others, challenges of personal and information privacy arise. Additionally, visual interference between screen content and the environment behind the display may decrease users' performance. We propose a solution by combining two transparent displays with a transparency-controlled backlight. The backlight, a piece of light guiding acrylic with edge-mounted LEDs, is opaque when turned on and transparent when off. This allows users to change the transparency of their displays ondemand and continuously, therefore controlling privacy and potential visual interference. Users can share contents instantaneously and at the same time have eye contact with their collaboration partner by interacting with the system using simple hand gestures.

\section{Technology}

For our system, we use two 22" transparent LCDs (Samsung LTI220MT02). To control the transparency of the setup as well as to increase contrast of the screen content, we add an additional layer of light-guiding acrylic (Evonik ACRYLITE® LED) with brightness controlled LED strips around the edges in-between the displays, serving as a transparent backlight. When the brightness of the LEDs is increased, the light of the LEDs is reflected inside the acrylic and the opaqueness of the backlight is increased, resulting in an opaque display for full brightness. When the LEDs are turned off and the screen content is faded out, the display is transparent. To provide users with the possibility to see through the display but still retain a private area, we split the backlight into two halves (vertically) and let users control each half separately. An optical half-wave retardation film (APHW92-003-PC$280 \mathrm{NM}$ ) is added to adjust the polarization between the two LCDs.

Permission to make digital or hard copies of part or all of this work for personal or classroom use is granted without fee provided that copies are not made or distributed for commercial advantage and that copies bear this notice and the full citation on the first page. Copyrights for third-party components of this work must be honored. For all other uses, contact the Owner/Author.

SIGGRAPH 2014, August 10 - 14, 2014, Vancouver, British Columbia, Canada.

2014 Copyright held by the Owner/Author.

ACM 978-1-4503-2961-3/14/08

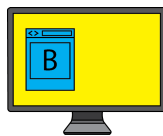

(a)

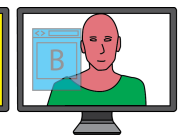

(b)

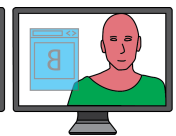

(c)

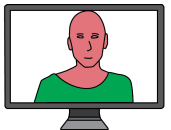

(d)

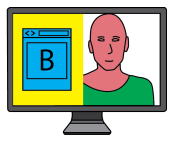

(e)
Figure 2: Possible states of the system: (a) opaque, (b) transparent screen content visible, (c) transparent screen content flipped, and (d) communication. Backlight is on in state (a). Additionally, the two halves of the screen can be controlled separately (e).

\section{Interaction}

Users can control the transparency of the display and easily switch between collaborative and private mode. The system provides the following states to users: (a) opaque for private work, (b) transparent with content visible for quick communication, (c) transparent with flipped content for sharing, and (d) completely transparent for longer discussions. Users can toggle transparency by making a vertical swipe gesture (up $=$ transparent, down $=$ opaque) (see Figure 1, left). Users can change transparency continuously by holding their hand statically (dwell time $300 \mathrm{~ms}$ ) in front of the display and then lifting (increase transparency) or lowering (decrease transparency) their hand. The dwell time allows us to distinguish between toggling and continuous change while being keeping the gesture consistent. With these interactions, transparency of the display and the screen content is changed and users can communicate with their partner without exposing their screen content. Additionally, users can control the two halves of the display separately by performing the gesture in front of the corresponding display half or centered to control both halves simultaneously. Users can easily and effortlessly share screen content like images, windows or other on-screen objects with their face-to-face partners, and flip contents with horizontal swipe gestures to avoid mirroring problems (see Figure 1, right).

\section{References}

ISHII, H., AND KoBAyAshi, M. 1992. ClearBoard: A Seamless Medium for Shared Drawing and Conversation with Eye Contact. In Proceedings of ACM CHI.

Lee, J., Olwal, A., IshiI, H., And Boulanger, C., 2013. SpaceTop: Integrating 2D and Spatial 3D Interactions in a Seethrough Desktop Environment. In Proceedings of ACM CHI. 